\title{
Dielectric breakdown in underoxidized magnetic tunnel junctions: Dependence on oxidation time and area
}

\author{
J. Ventura \\ IFIMUP and \\ Physics Department \\ University of Porto \\ Porto, Portugal \\ Email: joventur@fc.up.pt
}

\author{
R. Ferreira \\ INESC-MN and \\ Physics Department \\ IST \\ Lisbon, Portugal \\ Email: rferreira@inesc-mn.pt
}

\author{
J. B. Sousa \\ IFIMUP and \\ Physics Department \\ University of Porto \\ Porto, Portugal \\ Email: jbsousa@fc.up.pt
}

\author{
P. P. Freitas \\ INESC-MN and \\ Physics Department \\ IST \\ Lisbon, Portugal \\ Email: pfreitas@inesc-mn.pt
}

\begin{abstract}
Magnetic tunnel junctions (MTJs) with partially oxidized $9 \AA \mathrm{AlO}_{x}$-barriers were recently shown to have the necessary characteristics to be used as magnetoresistive sensors in high-density storage devices. Here we study dielectric breakdown in such underoxidized magnetic tunnel junctions, focusing on its dependence on tunnel junction area and oxidation time. A clear relation between breakdown mechanism and junction area is observed for the MTJs with the highest studied oxidation time: samples with large areas fail usually due to extrinsic causes (characterized by a smooth resistance decrease at dielectric breakdown). Small area junctions fail mainly through an intrinsic mechanism (sharp resistance decrease at breakdown). However, this dependence changes for lower oxidation times, with extrinsic breakdown becoming dominant. In fact, in the extremely underoxidized magnetic tunnel junctions, failure is exclusively related with extrinsic causes, independently of MTJ-area. These results are related with the presence of defects in the barrier (weak spots that lead to intrinsic breakdown) and of metallic unoxidized $\mathrm{Al}$ nanoconstrictions (leading to extrinsic breakdown).
\end{abstract}

Magnetic tunnel junctions (MTJs) consisting of two ferromagnetic (pinned and free) layers separated by an insulating barrier are the new generation of magnetoresistive sensors in high-density storage devices currently approaching 200-400 $\mathrm{Gbit} \mathrm{in}^{2}$ [1]. For MTJs to be implemented in hard drives, they must have low resistance-area product $\left(\mathrm{R} \times \mathrm{A}<1 \Omega \mu \mathrm{m}^{2}\right)$ and reasonable tunnel magnetoresistance (TMR $>20 \%)$ [2]. Such goals can be obtained in ultrathin tunnel junctions (insulating barrier thickness $t \sim 5-6 \AA$ ). However, this approach may lead to the presence of pinholes across the insulating barrier (regions of direct contact between the pinned and free layers having Ohmic resistance), resulting in an undesirably large interlayer coupling field. Similar TJ-characteristics (high TMR, low $\mathrm{R} \times \mathrm{A}$ ) were recently obtained by only partially oxidizing thicker $(9 \AA) \mathrm{AlO}_{x}$-barriers [3]. Although decreasing oxidation time lead to the decrease of both TMR and $\mathrm{R} \times \mathrm{A}$, fairly high TMR $\left(\sim 20 \% ; \mathrm{R} \times \mathrm{A} \sim 2-5 \Omega \mu \mathrm{m}^{2}\right)$ was still observed even when the oxidation was performed with the shutter closed.

Dielectric breakdown (DB) is the main reliability concern in MTJs [4]-[9] where it can occur through two distinct mech- anisms [5], [8]. Intrinsic breakdown occurs in MTJs with well formed oxide layers due to the action of the applied electrical field. This leads to an abrupt decrease of the TJ-electrical resistance (and TMR) as a consequence of the formation of microscopic ohmic shorts in the barrier [4], [10]. On the other hand, extrinsic breakdown is related with the growth of existing pinholes in the tunnel barrier due to localized heating caused by high electrical current densities flowing along such pinholes [6]. In experiments, this mechanism is characterized by a gradual variation of the electrical resistance at the onset of breakdown. Because tunnel junctions with thicker barriers have a lower concentration of pinholes, they fail intrinsically more often than thinner ones [8].

Here we study dielectric breakdown in underoxidized $\mathrm{CoFeB} / \mathrm{AlO}_{x} / \mathrm{CoFeB}$ MTJs. Breakdown in these samples occurs at localized spots of the barrier due to the extrinsic or intrinsic mechanisms, depending on both TJ-area and oxidation time. For MTJs with the largest studied oxidation time failure occurs more often due to extrinsic than intrinsic causes in junctions with large areas $\left(A \sim 10 \mu \mathrm{m}^{2}\right)$. On the other hand, MTJs with small areas $\left(A \sim \mu \mathrm{m}^{2}\right)$ are observed to fail mainly by an intrinsic mechanism. Furthermore, with decreasing oxidation time, extrinsic breakdown becomes the dominant failure mechanism, independently of MTJ-area. We further observe that extrinsic breakdown is usually preceded by intense Joule heating caused by large current densities flowing across unoxidized, metallic Al.

We studied several series of ion beam deposited magnetic tunnel junctions with different oxidation times [3]. The structure of the tunnel junctions studied was: glass/Al $(70 \AA) / T a$ $(90 \AA) / \mathrm{NiFe}(70 \AA) / \mathrm{CoFeB}(50 \AA) / \mathrm{AlO}_{x}(9 \AA) / \mathrm{X} / \mathrm{MnIr}(250$ $\AA) / \mathrm{Ta}(90 \AA)$, where $X$ is either an amorphous $\mathrm{CoFeB}(40$ $\AA)$ single layer [11] or a $\mathrm{CoFeB}(40 \AA) / \mathrm{Ru}(6 \AA) / \mathrm{CoFeB}$ $(40 \AA)$ synthetic antiferromagnet (SAF) structure [12]. The junctions were patterned to a rectangular shape with areas between $1 \times 1 \mu \mathrm{m}^{2}$ and $3 \times 8 \mu \mathrm{m}^{2}$. The $\mathrm{AlO}_{x}$ barrier was formed by a remote $\mathrm{Ar} / \mathrm{O}_{2}$ plasma $(110 \mathrm{~W} \mathrm{RF}$ in a $20 \mathrm{~cm}$ diameter assist ion gun) [3]. Ions drift to the chamber due 
to pressure gradient only. The oxidation is divided into three stages with total oxidation time $\left(t_{1}\right)+\left(t_{2}\right)+t_{3}$. During the first two stages $\left(t_{1}\right)+\left(t_{2}\right)$ the sample is protected by a shutter preventing most of the oxygen from reaching the sample. The plasma $\mathrm{O}_{2}$ content is progressively increased: in the first stage the plasma is created with $4 \mathrm{sccm}(\mathrm{Ar})+20 \mathrm{sccm}\left(\mathrm{O}_{2}\right)$ at a pressure $P=6.5 \times 10^{-5}$ Torr and in the next two stages one has $4 \mathrm{sccm}(\mathrm{Ar})+40 \mathrm{sccm}\left(\mathrm{O}_{2}\right)$ at $1.4 \times 10^{-4}$ Torr. The oxidation time of the studied series of MTJs ranged from $\left(25^{\prime \prime}\right)+\left(00^{\prime \prime}\right)+0^{\prime \prime}$ to $\left(30^{\prime \prime}\right)+\left(30^{\prime \prime}\right)+5^{\prime \prime}$.

The dependence of the tunnel magnetoresistance on the applied electrical current $\operatorname{TMR}(I)$ and current-voltage $\mathrm{V}(I)$ characteristics were simultaneously measured with an automated wafer probe station. Measurements were performed as follows [13]: under the electrical current $I$ one measures the resulting voltage drop in the parallel $\left(\mathrm{V}_{P}\right)$ and antiparallel $\left(\mathrm{V}_{A P}\right)$ states. The same procedure is performed for $-I$. The current magnitude is then increased and the above set of measurements repeated.

In Fig. Пa) we observe the electrical resistance versus applied bias current of a $\mathrm{CoFe} / \mathrm{AlO}_{x} / \mathrm{CoFeB} / \mathrm{MnIr}$ MTJ with $\left(30^{\prime \prime}\right)+\left(30^{\prime \prime}\right)+5^{\prime \prime}$ oxidation time and $A=1 \times 1 \mu \mathrm{m}^{2}$. Increasing the magnitude of the applied electrical current leads to a sudden, sharp and irreversible R-decrease at $|I| \approx 32 \mathrm{~mA}$. This abrupt decrease is associated with the intrinsic breakdown of the studied sample [5], through the formation of a pinhole in the barrier. As $I$ is further increased, a new breakdown event is seen (at $|I| \approx 55 \mathrm{~mA}$ ), associated with the formation of a new pinhole.

Tunnel junctions of the same series but with larger area show a fairly different behavior when DB occurs. Figure 1 b) displays the obtained results for a MTJ with $A=2 \times 3 \mu \mathrm{m}^{2}$. In this case breakdown (at $|I| \approx 35 \mathrm{~mA}$ ), leads to a slight and gradual R (and TMR; not shown) decrease. This behavior is related with defect-driven extrinsic breakdown of the barrier reflecting the growth of existing pinholes [5], [6] likely through thermally assisted electromigration of metallic ions from the electrodes into the barrier [14], [15]. Three more breakdown events are visible at higher currents $(|I| \approx 50 \mathrm{~mA}$, $\approx 65 \mathrm{~mA}$ and $\approx 80 \mathrm{~mA}$ ), further reducing $\mathrm{R}$ and bringing TMR to zero. The new breakdown points are characterized by small but sharp R-decreases, followed by gradual ones and are here associated with the formation of new pinholes in the barrier [16] and subsequent current-induced pinhole growth.

Breakdown in these underoxidized MTJs likely occurs at localized weak-spots of the barrier, where a large concentration of defects (oxygen vacancies or nanoconstrictions of metallic Al due to the underoxidation of the barrier) exists. With increasing MTJ-area, such defects are more expected to appear, leading to the observed change in the breakdown mechanism from intrinsic to extrinsic. In fact, for the MTJs with $\left(30^{\prime \prime}\right)+\left(30^{\prime \prime}\right)+5^{\prime \prime}$ oxidation time, we observed that $63 \%$ of those with small area failed by intrinsic DB, while this number decreased to $20 \%$ for the large area MTJs.

Decreasing the MTJ-oxidation time leads to increased probability of failure through an extrinsic DB-mechanism for

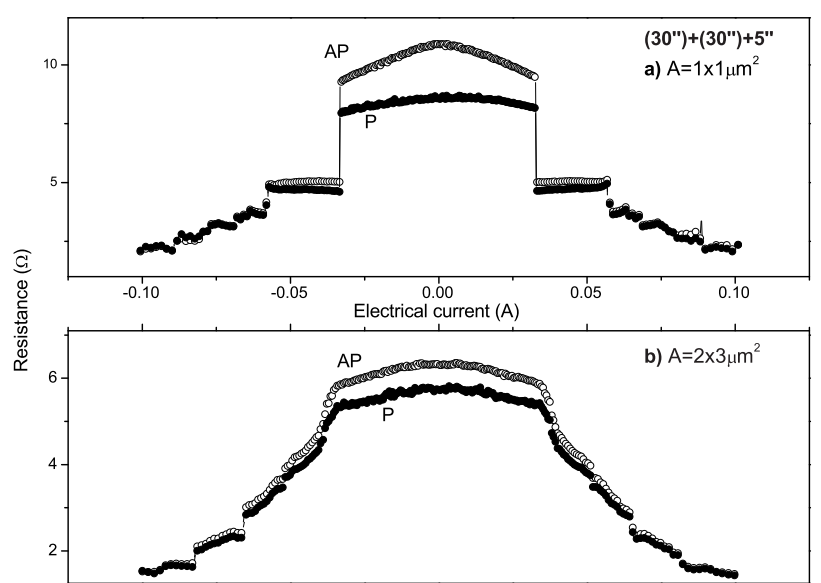

Fig. 1. Electrical resistance versus applied bias current of a MTJs with $\left(30^{\prime \prime}\right)+\left(30^{\prime \prime}\right)+5^{\prime \prime}$ oxidation time: (a) $A=1 \times 1 \mu \mathrm{m}^{2}$ and (b) $A=2 \times 3 \mu \mathrm{m}^{2}$, for both parallel (P) and antiparallel (AP) states. Notice the abrupt (smooth) $\mathrm{R}$ decrease observed at the intrinsic (extrinsic) breakdown of the barrier occurring for $A=1 \times 1 \mu \mathrm{m}^{2}\left(A=2 \times 3 \mu \mathrm{m}^{2}\right)$.

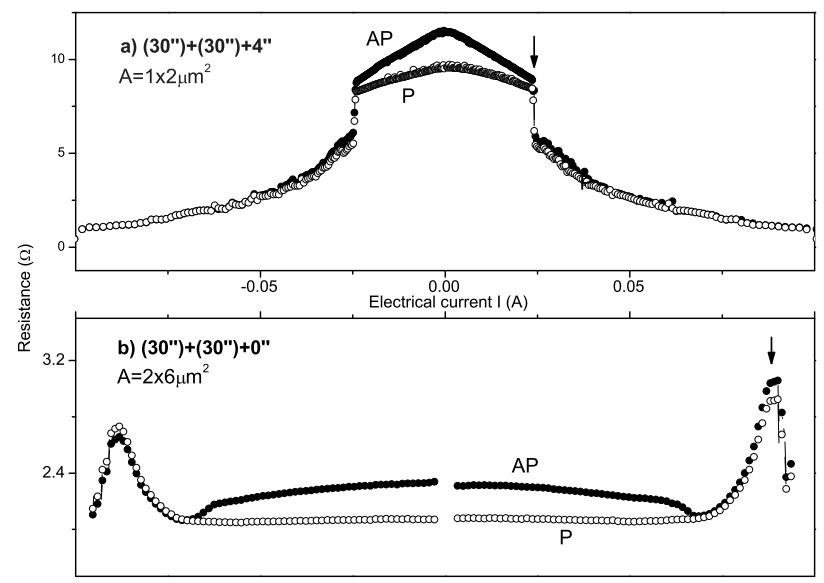

Fig. 2. Parallel and antiparallel electrical resistance versus applied current of MTJs with (a) $\left(30^{\prime \prime}\right)+\left(30^{\prime \prime}\right)+4^{\prime \prime}$ and (b) $\left(30^{\prime \prime}\right)+\left(30^{\prime \prime}\right)$ oxidation times.

both small and large area junctions, even though intrinsic breakdown is still observed [as displayed in Fig. 2] a) for a MTJ with $\left(30^{\prime \prime}\right)+\left(30^{\prime \prime}\right)+4^{\prime \prime}$ oxidation time]. Figure 2 b) shows electrical resistance versus bias current for a sample with $\left(30^{\prime \prime}\right)+\left(30^{\prime \prime}\right)+0^{\prime \prime}$ oxidation time, where extrinsic breakdown occurs (see arrow). Notice the fairly large R-increase just before TJ-dielectric breakdown, associated with heating due to large current densities flowing through metallic constrictions across the barrier (see below).

Tunnel junctions with extremely small oxidation time $\left[\left(25^{\prime \prime}\right)+\left(00^{\prime \prime}\right)+00^{\prime \prime}\right]$ all fail due to extrinsic reasons after large heating effects. Figure 3 displays TMR(I) and V(I) characteristics for a MTJ with $A=2 \times 4 \mu \mathrm{m}^{2}$ giving TMR $15 \%$. At $|I| \approx 30 \mathrm{~mA}$, TMR(I) sharply decreases. Corresponding $\mathrm{V}$ (I) characteristics for the parallel and antiparallel magnetic states (not shown) display a quasi-linear behavior also up to $|I| \approx 30 \mathrm{~mA}$. Fitting our data to Simmons' model gives 


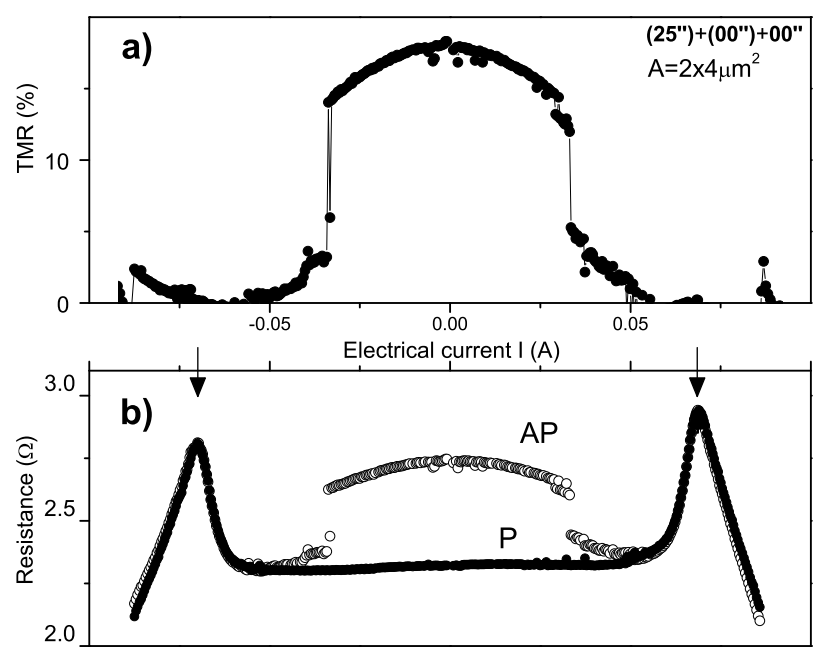

Fig. 3. (a) Tunnel magnetoresistance versus bias current $[\mathrm{TMR}(I)]$ and (b) corresponding $R(I)$ curves of a MTJ with $\left(25^{\prime \prime}\right)$ oxidation time.

the barrier thickness $t \approx 7 \AA$ and height $\varphi \approx 0.5 \mathrm{eV}$. The small values obtained indicate that only part of the initially deposited Al layer $(9 \AA)$ was oxidized and that the oxidized $\mathrm{Al}$ is likely not stequiometric. The observed decrease of TMR is due to the abrupt drop of the electrical resistance of the antiparallel state $\left(R_{\mathrm{AP}}\right)$. However, the electrical resistance of the parallel state $\left(R_{\mathrm{P}}\right)$ remains constant [Fig. 3 $\left.\left.\mathrm{b}\right)\right]$. Thus, this decrease is not related with junction breakdown but is likely due to heating (leading to the loss of exchange bias) or spin-torque driven instability of the antiparallel state (due to the high current densities flowing through unoxidized $\mathrm{Al}$; see below) [17]. At moderate applied electrical currents, we observe a significant R-increase [Fig. 3 b)], a behavior that we associate with Joule heating, due to high current densities flowing through metallic unoxidized $\mathrm{Al}$ nanoconstrictions. The existence of such metallic paths was indeed confirmed by measurements of the temperature dependence of the electrical resistance of MTJs of the same series (equal oxidation time) [13]. Only at higher electrical currents [see arrows in Fig. 3b)] is the electrical resistance seen to decrease, due to the extrinsic breakdown of the barrier. We conclude that nanoconstrictions of unoxidized $\mathrm{Al}$ are the features behind extrinsic dielectric breakdown in underoxidized MTJs.

Transport in these tunnel junctions then arises from two channels acting in parallel: one consisting of metallic paths of unoxidized $\mathrm{Al}$ (with resistance $\mathrm{R}_{m}$ ) and another of tunneling through the oxidized $\mathrm{AlO}_{x}$ (with resistance $\mathrm{R}_{t}$ ). We can estimate the area of the metallic conduction channels assuming that their electrical resistance is given by the Maxwell formulation $R_{m}=\frac{\rho}{2 a}$ [ $\rho$ the electrical resistivity of the constriction (assumed $\approx 10 \mu \Omega \mathrm{cm}$ ) and $a$ the radius of the metallic channel]. Considering $R_{m} \ll R_{t}$, we obtain for $R \approx 2.4 \Omega$ [Fig. [3 b)], $a \approx 200 \AA$, which corresponds to $\approx 0.004 \%$ of the total TJ-area. This is the upper limit of $a$ since the tunnelresistance arising from the oxidized part of the junction should also be included. The extremely small area of the unoxidized
Al leads to high local current densities and thus to the observed heating [18].

In summary, dielectric breakdown in underoxidized MTJs occurs at localized spots in the barrier, likely where a large concentration of defects exists. We observed a clear dependency of the breakdown process on the MTJ area: Failure in MTJs with large areas is usually of an extrinsic nature, while small area junctions fail mainly by an intrinsic mechanism. Nevertheless, with decreasing oxidation time, the extrinsic breakdown becomes dominant, independently of the MTJ-area. Extrinsic breakdown is preceded by Joule heating caused by large current densities flowing across unoxidized, metallic $\mathrm{Al}$ nanoconstrictions whose size was estimated.

\section{ACKNOWLEDGMENT}

Work supported in part by POCTI/CTM/59318/2004, IST2001-37334 NEXT MRAM and POCTI/CTM/36489/2000 projects. J. Ventura, and R. Ferreira are thankful for FCT grants (SFRH/BPD/21634/2005 and SFRH/BD/6501/2001).

\section{REFERENCES}

[1] S. Mao, Y. Chen, F. Liu, X. Chen, B. Xu, P. Lu, M. Patwari, H. Xi, C. Chang, B. Miller, D. Menard, B. Pant, J. Loven, K. Duxstad, S. Li, Z. Zhang, A. Johnston, R. Lamberton, M. Gubbins, T. McLaughlin, J. Gadbois, J. Ding, B. Cross, S. Xue, and P. Ryan, "Commercial TMR Heads for Hard Disk Drives: Characterization and Extendibility At 300 Gbit/in²", IEEE Trans. Magn., vol. 42, pp. 97-102, February 2006.

[2] M. Takagishi, K. Koi, M. Yoshikawa, M. Funayama, H. Iwasaki, and M. Sahashi, "The applicability of CPP-GMR heads for magnetic recording", IEEE Trans. Magn., vol. 38, pp. 2277-2282, September 2002.

[3] R. Ferreira, P. P. Freitas, M. MacKenzie, and J. N. Chapman, "Low resistance magnetic tunnel junctions prepared by partial remote plasma oxidation of $0.9 \mathrm{~nm} \mathrm{Al} \mathrm{barriers",} \mathrm{Appl.} \mathrm{Phys.} \mathrm{Lett.,} \mathrm{vol.} \mathrm{86,} \mathrm{pp.} \mathrm{192502,}$ July 2005.

[4] W. Oepts, H. J. Verhagen, R. Coehoorn, and W. J. M. de Jonge, "Analysis of breakdown in ferromagnetic tunnel junctions", J. Appl. Phys., vol. 86, pp. 3863-3872, October 1999.

[5] B. Oliver, G. Tuttle, Q. He, X. Tang, and J. Nowak, "Two breakdown mechanisms in ultrathin alumina barrier magnetic tunnel junctions", $J$. Appl. Phys., vol. 95, pp. 1315-1322, February 2004.

[6] B. Oliver, Q. He, X. Tang, and J. Nowak, "Dielectric breakdown in magnetic tunnel junctions having an ultrathin barrier", J. Appl. Phys., vol. 91, pp. 4348-4352, April 2002.

[7] D. Rao, K. Sin, M. Gibbons, S. Funada, M. Mao, C. Chien, and H.-C. Tong, "Voltage-induced barrier-layer damage in spin-dependent tunneling junctions", J. Appl. Phys., vol. 89, pp. 7362-7364, June 2001.

[8] K.-S. Kim, B. K. Cho, T. W. Kim, and W. J. Park, "Junction area dependence of breakdown characteristics in magnetic tunnel junctions", J. Appl. Phys., vol. 93, pp. 8364-8366, May 2003.

[9] J. Schmalhorst, H. Brückl, M. Justus, A. Thomas, G. Reiss, M. Vieth, G. Gieres, and J. Wecker, "Evolution of the dielectric breakdown in $\mathrm{Co} / \mathrm{Al}_{2} \mathrm{O}_{3} / \mathrm{Co}$ junctions by annealing", J. Appl. Phys., vol. 89, pp. 586589, January 2001.

[10] S. Lombardo, J. H. Stathis, B. P. Linder, K. L. Pey, F. Palumbo, and C. H. Tung, "Dielectric breakdown mechanisms in gate oxides", $J$. Appl. Phys., vol. 98, pp. 121301, December 2005.

[11] S. Cardoso, R. Ferreira, P. P. Freitas, M. MacKenzie, J. Chapman, J. O. Ventura, J. B. Sousa, and U. Kreissig, "Ferromagnetic coupling field reduction in $\mathrm{CoFeB}$ tunnel junctions deposited by ion beam", IEEE Trans. Magn., vol. 40, pp. 2272-2274, July 2004.

[12] M. Rickart, A. Guedes, B. Negulescu, J. Ventura, J. B. Sousa, P. Diaz, M. MacKenzie, J. N. Chapman, and P. P. Freitas, "Exchange coupling of bilayers and synthetic antiferromagnets pinned to MnPt", Eur. Phys. J. B, vol. 45, pp. 207-212, May 2005. 
[13] J. Ventura, J. Teixeira, Yu. G. Pogorelov, J. B. Sousa, R. Ferreira, and P. P. Freitas, "Spin-dependent two-level resistance fluctuations in underoxidized tunnel junctions", J. Appl. Phys., vol. 99, pp. 08T301, April 2006.

[14] J. Ventura, J. Araujo, J. B. Sousa, Y. Liu, Z. Zhang, and P. P. Freitas, "Nanoscopic processes of Current Induced Switching in thin tunnel junctions", IEEE Trans. Nanotechnol., vol. 5, pp. 142-148, March 2006.

[15] J. Ventura, J. B. Sousa, Y. Liu, Z. Zhang, and P. P. Freitas, "Electromigration in thin tunnel junctions with ferromagnetic/nonmagnetic electrodes: Nanoconstrictions, local heating, and direct and wind forces", Phys. Rev. B, vol. 72, pp. 094432, September 2005.

[16] W. Oepts, H. J. Verhagen, D. B. de Mooij, V. Zieren, R. Coehoorn, and W. J. M. de Jonge, "Observation and analysis of breakdown of magnetic tunnel junctions", J. Magn. Magn. Mater., vol. 198, pp. 164-166, June 1999.

[17] J. Grollier, V. Cros, H. Jaffrès, A. Hamzic, J. M. George, G. Faini, J. Ben Youssef, H. Le Gall, and A. Fert, "Field dependence of magnetization reversal by spin transfer", Phys. Rev. B, vol. 67, no. 17, pp. 174402, May 2003.

[18] J. Ventura, A. Pereira, J. M. Teixeira, J. P. Araujo, F. Carpinteiro, J. B. Sousa, Z. Zhang, Y. Liu, and P. P. Freitas, "Heat generation in tunnel junctions for current-written pinned layer switching", Mat. Sci. Forum, vol. 514-516, pp. 323-327, February 2006. 